\title{
Iatrogenic diaphragmatic hernia with fecopneumothorax following minimally invasive esophagectomy and liver resection
}

\author{
Ammara A. Watkins, MD, MPH, ${ }^{\mathrm{a}, \mathrm{b}}$ Aditya Kalluri, MD, PhD,${ }^{\mathrm{c}}$ Alok Gupta, MD, FACS, ${ }^{\mathrm{d}}$ and \\ Sidhu P. Gangadharan, MD, MHCM, ${ }^{\mathrm{c}}$ Boston, Mass
}

\footnotetext{
From the ${ }^{a}$ Division of Thoracic and Cardiovascular Surgery, Lahey Hospital and Medical Center, Burlington, Mass; ' Tufts Medical School, Boston, Mass; ' Division of Thoracic Surgery and Interventional Pulmonology, and ${ }^{\mathrm{d} D i v i s i o n}$ of Acute Care Surgery, Trauma, and Surgical Critical Care, Beth Israel Deaconess Medical Center, Harvard Medical School, Boston, Mass.

Disclosures: The authors reported no conflicts of interest.

The Journal policy requires editors and reviewers to disclose conflicts of interest and to decline handling or reviewing manuscripts for which they may have a conflict of interest. The editors and reviewers of this article have no conflicts of interest.

Received for publication Oct 22, 2021; accepted for publication Oct 26, 2021; available ahead of print Nov 3 , 2021.

Address for reprints: Ammara A. Watkins, MD, MPH, Division of Thoracic and Cardiovascular Surgery, Lahey Hospital and Medical Center, 41 Mall Rd., Burlington, MA 01805 (E-mail: ammara.watkins@lahey.org). JTCVS Techniques 2022;11:89-91

2666-2507

Copyright (C) 2021 The Author(s). Published by Elsevier Inc. on behalf of The American Association for Thoracic Surgery. This is an open access article under the CC BY-NC-ND license (http://creativecommons.org/licenses/bync-nd/4.0/)

https://doi.org/10.1016/j.xjtc.2021.10.052
}

Video clip is available online.

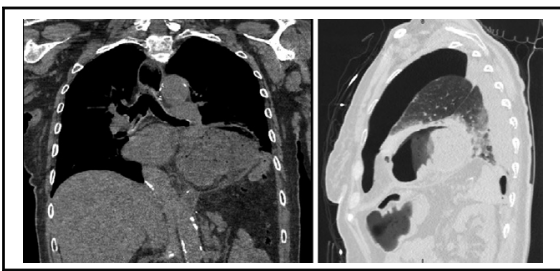

CT exhibiting a diaphragmatic defect, separate from the conduit's course, and pneumothorax.

\section{CENTRAL MESSAGE \\ Diaphragmatic herniation may occur after esophagectomy and liver resection. We describe the management of fecopneumo- thorax secondary to a diaphrag- matic defect caused by liver mobilization.}

See Commentaries on pages 92 and 94 .

\section{CLINICAL SUMMARY}

A 65-year-old woman underwent neoadjuvant chemoradiation and minimally invasive Ivor-Lewis esophagectomy (MIE) for esophageal adenocarcinoma. A restaging positron emission tomography scan showed regression of tumor avidity and no metastasis. Nevertheless, during her MIE, a $3-\mathrm{cm}$ liver lesion was noted in the lateral aspect of segment II. The left triangular ligament was divided and the liver lesion was excised using the Enseal (Ethicon Inc) device. Final pathology of the esophageal specimen was T1b N0 esophageal adenocarcinoma. The liver lesion revealed a focus of resolving hepatic injury with nodular regenerative hyperplasia, possibly secondary to neoadjuvant treatment, with no malignancy seen.

Four years later, she presented with new left shoulder pain, A chest radiograph (CXR) and shoulder film was read as normal. Retrosternal air was mischaracterized as her gastric conduit, her shoulder pain was attributed to arthritis, and she was discharged from the emergency room with pain medication. She represented 3 days later with respiratory distress, renal failure, and lactic acidosis. CXR demonstrated a left-sided pneumothorax, intrathoracic bowel, and mediastinal shift. A computed tomography (CT) scan showed a left diaphragmatic defect lateral to the conduit's course, with herniation of transverse colon into the chest (Figure 1). A chest tube was placed which drained feculent fluid and air. She was immediately taken to the operating room.

After placement of a double lumen tube, the patient was positioned in right lateral decubitus and angled slightly posterior as one would do for a thoracoabdominal incision. This allowed access for both thoracotomy and midline laparotomy. A left posterolateral thoracotomy was performed in the fifth interspace. Frank stool was removed and the lung was mobilized. A necrotic and perforated loop of transverse colon was protruding through a $3-\mathrm{cm}$ defect in the central tendon of the left diaphragm (Video 1). This diaphragmatic defect was distinctly separate from the hiatal opening. We closed a small perforation in the colon primarily to prevent 


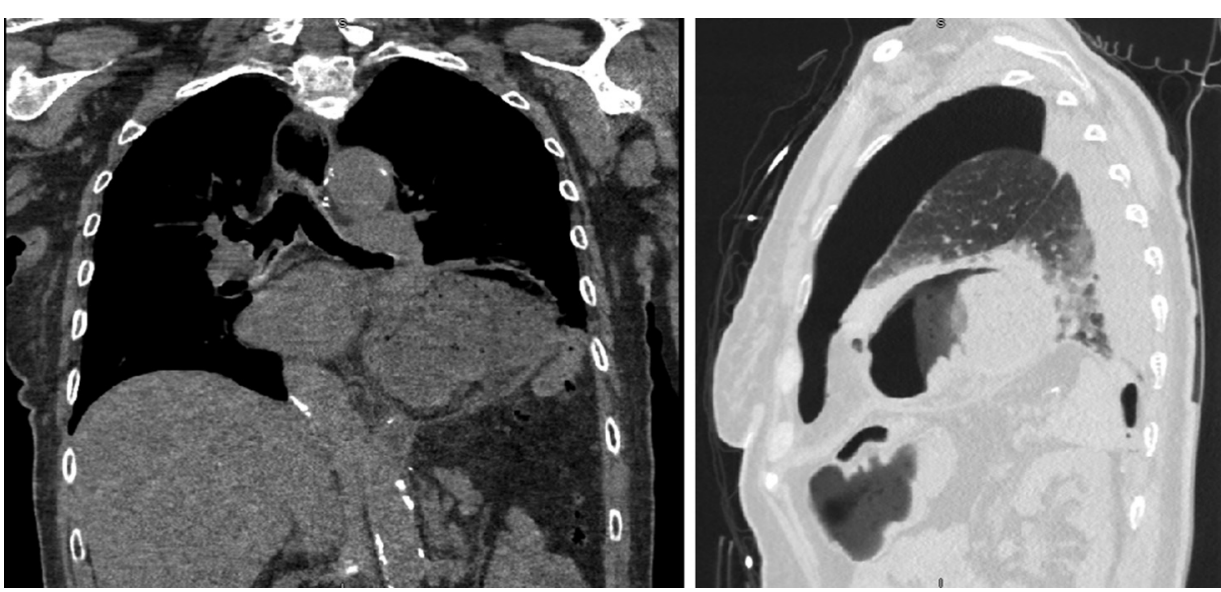

FIGURE 1. CT exhibiting a diaphragmatic defect, separate from the conduit's course, and pneumothorax.

additional spillage while the pleural space was washed out and debrided. An attempt was made to pull additional colon into the chest for repair; however, this was not possible due to tethering of abdominal colonic attachments. A midline laparotomy was then performed, and the diaphragmatic defect was enlarged to enable delivery of the strangulated loop of transverse colon back into the peritoneal cavity. The diaphragmatic defect was then closed with a tensionfree closure utilizing figure-of- 8 Prolene sutures.

Upon laparotomy there was no contamination noted within the abdomen. Pan-diverticulosis was noted but not present in the perforated section, with the nearest diverticulum $10 \mathrm{~cm}$ proximal to the perforation. In the absence of local diverticular disease and contamination, a segmental colon resection with stapled anastomosis was performed. The inside of the chest was scrubbed and irrigated with povidone-iodine and widely drained with 3 chest tubes.

The patient had a 7-week inpatient postoperative course and wound complications requiring vacuum therapy, but ultimately returned home independently and remains disease-

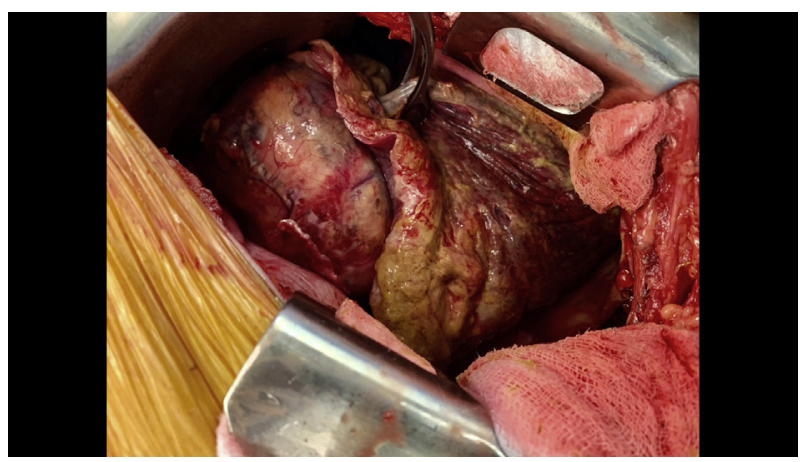

VIDEO 1. Presentation and operative management of fecopneumothorax utilizing left thoracotomy, midline laparotomy, segmental colon resection, and primary diaphragm repair. Video available at: https://www.jtcvs.org/ article/S2666-2507(21)00746-X/fulltext. free 1 year following her reoperation. This case report was institutional review board exempt and the patient provided informed consent for publication.

\section{DISCUSSION}

Diaphragmatic herniation is a known complication following both esophagectomy and liver resection. ${ }^{1,2}$ The majority of diaphragmatic herniations following MIE are paraconduit hernias, which occur alongside the conduit, typically between the left crus and the conduit. ${ }^{3}$ Rarely, fecopneumothorax can result from paraconduit herniation. ${ }^{4}$ Unlike the typical location of paraconduit herniation, we report a diaphragmatic herniation following MIE that was separate from the conduit's hiatus resulting in fecopneumothorax and profound morbidity. It is suspected that this diaphragmatic defect was secondary to liver mobilization and resection done at the time of her esophagectomy. We believe a small tear from liver mobilization undetected at the time of her initial MIE widened over time as the diaphragm continued to remodel and weaken. Additionally, the unavoidable pressure differential between the abdomen and chest eventually contributed to colonic herniation into the left chest and the creation of a chronic traumatic diaphragmatic hernia. ${ }^{5}$ The colon became strangulated and ischemic, resulting in intrathoracic perforation.

This case demonstrates the importance of considering diaphragmatic herniation in any patient with previous esophagectomy or liver mobilization. Although the most common complaint for paraconduit hernias is abdominal pain, dysphagia, constipation, chest pain, shortness of breath, and shoulder pain are also described. ${ }^{3,6}$ As with this patient, symptoms can be atypical, and the diagnosis delayed. CXR has low sensitivity in diagnosing postesophagectomy diaphragmatic hernias. Even when air fluid levels or retrocardiac air is present, these can be normal findings after an Ivor-Lewis esophagectomy and can be mistaken 
for the conduit. CT is the gold standard and should be pursued in any postesophagectomy patients who present with new chest symptoms, even if nonspecific. A high index of suspicion is needed to promptly diagnose and manage these patients.

\section{References}

1. Oor JE, Wiezer MJ, Hazebroek EJ. Hiatal hernia after open versus minimally invasive esophagectomy: a systematic review and meta-analysis. Ann Surg Oncol. 2016;23:2690-8
2. Esposito F, Lim C, Salloum C, Osseis M, Lahat E, Compagnon P, et al. Diaphragmatic hernia following liver resection: case series and review of the literature. Ann Hepatobiliary Pancreat Surg. 2017;21:114-21.

3. Kent MS, Luketich JD, Tsai W, Churilla P, Federle M, Landreneau R, et al. Revisional surgery after esophagectomy: an analysis of 43 patients. Ann Thorac Surg. 2008;86:975-83.

4. Markogiannakis H, Theodorou D, Tzertzemelis D, Dardamanis D, Toutouzas KG, Misthos P, et al. Fecopneumothorax: a rare complication of esophagectomy. Ann Thorac Surg. 2007;84:651-2.

5. Blitz M, Louie BE. Chronic traumatic diaphragmatic hernia. Thorac Surg Clin. 2009; 19:491-500.

6. Lee J, Nam SH, Kim SW, Hong JM, Kim D. Diaphragmatic hernia with isolated shoulder pain evoked by surfeit. Ann Transl Med. 2019;7:11. 